\title{
Rapid detection of low-abundance K-ras mutation in stools of colorectal cancer patients using chip-based temperature gradient capillary electrophoresis
}

\author{
Huidan Zhang, Xiaonan Wang, Qian Ma, Zhe Zhou and Jin Fang*
}

\begin{abstract}
Mutant $K$-ras provides an independent negative predictive marker for epidermal growth factor receptor (EGFR)-targeted therapy in colorectal cancers (CRCs). Rapid, sensitive, and cost-effective screening for $K$-ras status will overarch rational personalized medicine. Stool-based DNA testing offers unique advantages for CRC screening such as noninvasiveness, high specificity, and patient compliance, whereas complicated procedures and the low sensitivity of the present approaches have hampered its application on a wide scale. In this study, a chip-based temperature gradient capillary electrophoresis (TGCE) technique was applied to detect low-abundance $K$-ras mutations under a pooled experiment and analyze $K$-ras mutations in 30 paired stool samples and cancer tissues of CRC patients and 15 stool samples of healthy volunteers. The chip-based TGCE results showed that the successful analysis of $K$-ras status could be achieved within 6 min with an extremely low sample consumption of $14 \mathrm{nl}$. Detection is sensitive enough to reliably report $0.2 \%$ mutant CRC cells in a wild-type background, and $0.5 \mathrm{ng}$ of template DNA was sufficient for chip-based TGCE. Of the 30 stool samples of CRC patients analyzed, 17 (57\%) harbored K-ras mutations, and the lowest percentage of the detectable mutant $K$-ras in stool samples was $2 \%$. The coincidence rate for $K$-ras mutations between stools and tissues obtained by the chip-based method reached $97 \%$ (29/30). One of the 15 stool samples of normal controls carried K-ras mutations, producing a specificity of $93 \%$. Clone sequencing data entirely confirmed the results obtained by chip-based TGCE. The study demonstrates that chip-based TGCE is capable of rapidly screening low-abundance $K$-ras mutations with high sensitivity, reproducibility, simplicity, and significant savings of time and sample. Application of this method to genotype the $K$-ras gene in stools would provide a potential means for predicting the effectiveness of EGFR-targeted therapy in CRC patients using noninvasive approaches.
\end{abstract}

Laboratory Investigation (2011) 91, 788-798; doi:10.1038/labinvest.2010.200; published online 17 January 2011

KEYWORDS: colorectal cancer; K-ras; microfluidic chip; mutation detection; stool; temperature gradient capillary electrophoresis (TGCE)

Epidermal growth factor receptor (EGFR) is a receptor tyrosine kinase that controls cell growth, differentiation, and transformation, and is highly expressed in most common human cancers. It has been reported that EGFR expression is detected in up to $80 \%$ of colorectal cancer (CRC) patients and is associated with metastasis and reduced survival rates. ${ }^{1}$ Therefore, several therapeutic agents that target EGFR, such as cetuximab and panitumumab, were developed for CRC treatment and significantly improved the survival rate and living quality of patients. ${ }^{2,3}$ The $K$-ras gene-encoded $21-\mathrm{kD}$ protein, a key downstream effector of EGFR, has a critical role in controlling signal transduction pathways during cell growth. Constitutively activating mutations in $\mathrm{K}$-ras, particularly at 'limited hot spot' codons 12 and 13, may lead to uncontrolled cell proliferation and may render tumor cells independent of EGFR signaling and thereby resistant to EGFR-targeted therapies. ${ }^{4-6}$ An American Society of Clinical Oncology (ASCO) provisional clinical opinion (PCO) has addressed the utility of $K$-ras gene mutation testing in patients with metastatic CRC to predict the response to antiEGFR therapy with cetuximab or panitumumab. ${ }^{7}$ Therefore, it is of the utmost importance to screen and assess these

Department of Cell Biology, Key Laboratory of Cell Biology, Ministry of Public Health, and Key Laboratory of Medical Cell Biology, Ministry of Education, China Medical University, Shenyang, China

${ }^{*}$ Correspondence: Professor J Fang, PhD, Department of Cell Biology, Key Laboratory of Cell Biology, Ministry of Public Health, and Key Laboratory of Medical Cell Biology, Ministry of Education, China Medical University, 92 Beier Road, Heping District, Shenyang 110001, China.

E-mail: jfang61@netease.com or jfang61@gmail.com

Received 10 May 2010; revised 15 November 2010; accepted 23 November 2010 
patients for $K$-ras status to guide personalized medicine and to avoid the application of costly drugs that would only cause toxic side effects.

In the present screening strategies, stool-based DNA testing for CRC may offer unique advantages over other kinds of clinical materials such as cancer tissues because of noninvasiveness, high specificity, and patient compliance. ${ }^{8-10}$ However, the proportion of genes derived from cancer cells in stools is as low as $1 \% .{ }^{11}$ At this concentration, mutations in stool DNA will not be readily detected using the most currently available gene analyzing methods, ${ }^{12}$ such as restriction fragment length polymorphism (RFLP), ${ }^{13}$ single-strand conformation polymorphism (SSCP), ${ }^{14}$ and microarray chips. ${ }^{15}$ The major drawbacks of these assays are that they lack sufficient sensitivity and high stability, and they are rather time and sample consuming and expensive for use in screening for molecular diagnostic purposes. Therefore, a rapid, sensitive, and economical method for clinical detection of $K$-ras genotypes in stools is in great demand. In recent years, most of the slab gel-based techniques for the detection of nucleotide mutations have been developed and implemented in capillary electrophoresis (CE) systems, with significant improvements in sensitivity and detection speed. ${ }^{16}$ Temperature gradient capillary electrophoresis (TGCE) is a mutation screening technology that was developed by transplanting temperature gradient gel electrophoresis (TGGE) to a CE platform, in which DNA fragments consisting of homoduplexes and heteroduplexes are separated according to the different melting temperatures $(\mathrm{Tm}){ }^{17}$ During electrophoretic separation, the sharply retarded mobility of heteroduplexes facilitates the achievement of highresolution efficiency for even a single nucleotide change. It is superior to many of the previously listed CE-based presequencing screening techniques. Another outstanding advantage of TGCE is its ability to analyze a wide variety of mutations without knowing their exact nature beforehand, which allows the detection of all base changes in the target DNA fragments. ${ }^{16,18,19}$ As for the $K$-ras gene studied here, both codons 12 and 13 that contribute to directing chemotherapy can also be detected simultaneously.

Conventional TGCE requires at least $10 \%$ of the mutant DNA in a wild-type DNA background, but it is not sufficiently sensitive for the detection of $\mathrm{K}$-ras mutations in cancer cells within stool samples. ${ }^{20}$ The efficiency of detecting genetic variants can be much improved when the electrophoretic process is transplanted onto the microfluidic chip, a new microanalysis system with notable advantages involving higher sensitivity, increased speed, and reduced sample/reagent consumption. ${ }^{21,22}$ Buch et al ${ }^{23}$ first fabricated a microfluidic device, employing TGCE to rapidly achieve the successful detection of model single-nucleotide polymorphism (SNP) samples. However, the large external heater assemblies in their spatial format increased difficulty with respect to accuracy control and miniaturization. ${ }^{23}$ In our previous work, a chip-based CE platform integrated with a slantwise radiative heating system that was able to establish spatial temperature gradient using simple peripheral equipment and manipulation was developed for very simple, reliable, and cost-effective DNA mutation detection by chipbased TGCE. Various SNP samples and $K$-ras gene mutations in six colon cancer cell lines were successfully detected rapidly within $6 \mathrm{~min}^{24}$ In the present study, we further explore the possibility of chip-based TGCE analysis for the detection of low-abundance $K$-ras mutations and the feasibility for genotyping the $K$-ras gene in DNA extracted from stools to perform screening and predict the effectiveness of EGFRtargeted therapy in patients with CRC.

\section{MATERIALS AND METHODS Cell Lines and Clinical Samples}

Human CRC cell lines SW480 and HT29 were cultivated at $37^{\circ} \mathrm{C}$ with $5 \% \mathrm{CO}_{2}$ in DMEM culture medium supplemented with $10 \%$ fetal bovine serum. A total of 30 naturally evacuated stool samples from CRC patients were collected on the defecation day $>2$ weeks after colonoscopy and biopsy. The stool sample of each patient was aliquoted into several equal units of $\sim 150 \mathrm{mg}$. Aliquots were placed in the $2 \mathrm{ml}$ freezing tube and then immersed in liquid nitrogen. In addition, 30 paired primary CRC tissues were stored in a $-80{ }^{\circ} \mathrm{C}$ freezer until analyzed. The study complied with approved institutional guidelines regarding patient information and consent. None of the patients underwent preoperative radiotherapy and chemotherapy, and all gave informed consent for our study. The characteristics of the patients are summarized in Table 1 . The median age of the patients was 58 years (range 32-74 years). There were 19 male and 11 female patients. The tumors were located in the colons in 17 patients and in the recta in 13 patients. The clinical stage of the patients according to Dukes' classification was as follows: stage $A$ in 6 patients, stage $B$ in 4 patients, stage $C$ in 9 patients, and stage $\mathrm{D}$ in 11 patients. As a normal control, 15 stool samples were obtained from healthy volunteers who had no evidence of malignant tumors on colonoscopy, gastroduodenoscopy, and upper abdominal ultrasonography. As shown in Table 1, the median age of the healthy volunteers was 56 years (range $45-75$ years). There were 9 males and 6 females.

\section{DNA Extraction and PCR Amplification}

Genomic DNA was extracted from cell lines and frozen CRC tissues using a QIAamp DNA mini kit, and genomic DNA from the paired stool samples was isolated using a QIAamp DNA Stool mini kit. To detect $K$-ras point mutations at codons 12 and 13 (exon 1), a 293-bp fragment was amplified by PCR using the following primers: $5^{\prime}$-GTACTGGTGGAG TATTTGATAGTG- $3^{\prime}$ (forward) and $5^{\prime}$-AAAGAATGGTC CTGCACCAGTAATA- $3^{\prime}$ (reverse). The forward primer was synthesized with a 40-bp GC-rich region at the $5^{\prime}$ end to artificially introduce a GC-clamp (5'-CGCCCGCCGCGCCC CGCGCCCGTCCCGCCGCCCCCGCCCG- $\left.3^{\prime}\right) .{ }^{17}$ PCR amplification was performed in $25 \mu \mathrm{l}$ reaction mixture containing 
Table 1 Characteristics of patients and healthy volunteers

\begin{tabular}{lcc}
\hline Characteristic & Patients & Healthy volunteers \\
\hline Number of individuals & 30 & 15 \\
Age, years & & 55.8 \\
Mean & 56.5 & $56(45-75)$ \\
Median (range) & $58(32-74)$ & \\
Sex & & 6 \\
Male & & \\
Female & 19 & \\
Tumor site & 11 & \\
Colon & & \\
Rectum & & \\
Stage & 17 & \\
A & 13 & \\
B & & \\
C & & \\
D & & \\
\hline
\end{tabular}

$0.2 \mathrm{mM}$ dNTPs, $0.5 \mu \mathrm{M}$ of each primer, $1 \times$ PCR buffer (10 mM Tris- $\mathrm{HCl}, \mathrm{pH} 8.3,1.5 \mathrm{mM} \mathrm{MgCl}_{2}$, and $50 \mathrm{mM} \mathrm{KCl}$ ), and 2.5 U Pyrobest DNA polymerase. The cell line and tissue/ stool genomic DNA $(1 \mu \mathrm{l})$ were used as templates. The PCR was performed with preheating at $95^{\circ} \mathrm{C}$ for $5 \mathrm{~min}$, followed by 35 cycles of $94{ }^{\circ} \mathrm{C}$ for $1 \mathrm{~min}, 55^{\circ} \mathrm{C}$ for $1 \mathrm{~min}, 72^{\circ} \mathrm{C}$ for $2 \mathrm{~min}$, and a final extension at $72{ }^{\circ} \mathrm{C}$ for $10 \mathrm{~min}$.

\section{Instrument Fabrication}

A crossed-channel glass chip with a 1.0-cm-long sample loading channel and a $4.5-\mathrm{cm}$ separation channel from the crossing to the outlet reservoir $(5.0 \mathrm{~cm}$ total length) were fabricated by wet etching and thermal bonding as described in Jia et al. ${ }^{25}$ A laboratory-assembled chip-based CE system with a confocal laser-induced fluorescence (LIF) detection device described elsewhere and equipped with a 473-nm diode laser as an excitation source was used in this work. ${ }^{26} \mathrm{~A}$ home-built four-output $1500 \mathrm{~V}$ programmable power supply was used for on-chip sample injection and electrophoretic separation. The fluorescence signal was recorded by a chart recorder. The slantwise radiative heating system-based TGCE system in the microfluidic chip was set up for DNA mutation analysis as shown in Figure 1. A homemade thermostated heater plate consisting of an aluminum plate and a semiconductor heating element was slightly slanted relative to the plane of the glass chip by inserting a thin spacer between the plate and chip at the upstream end of the separation channel.

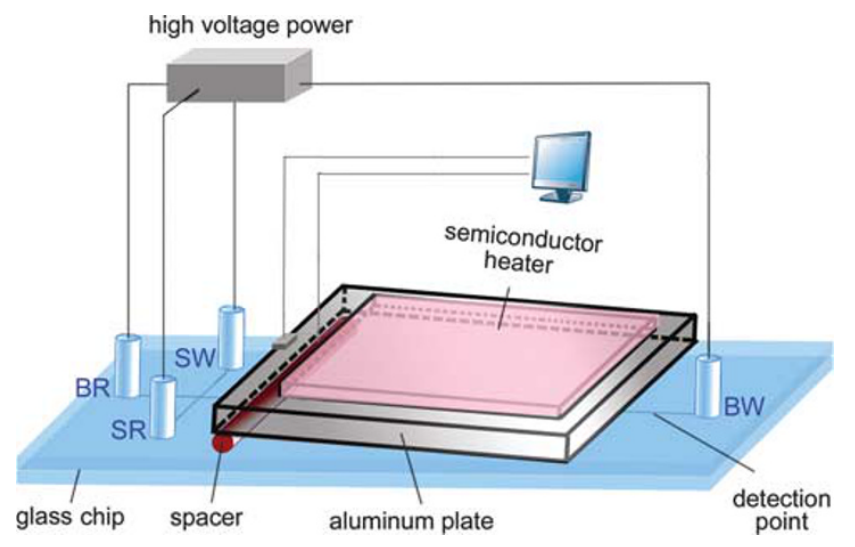

Figure 1 Schematic view of chip-based TGCE system. SR: sample reservoir; BR: buffer reservoir; SW: sample waste reservoir; BW: buffer waste reservoir.

The temperature was raised to a set temperature by a computer program written in LabVIEW 6.0. A Pt100 sensor chip epoxyed to the top of the aluminum plate maintained the set temperature by feedback-controlling the heating voltage. Owing to differences in radiative heating along the $3-\mathrm{cm}$ length of the heating region, a continuous and uniform spatial temperature gradient formed.

\section{Analysis for Mutation by Chip-Based TGCE}

GC-clamped PCR amplicons from cell lines and clinical samples were denatured and reannealed to form heteroduplexes. Crude PCR products were used without further purification. The denaturing and reannealing procedure is as follows: the temperature was held for $3 \mathrm{~min}$ at $95^{\circ} \mathrm{C}$, ramped from 95 to $25^{\circ} \mathrm{C}$ at a rate of $2{ }^{\circ} \mathrm{C}$ per min, held for 3 min at $25^{\circ} \mathrm{C}$, and finally ramped from 25 to $4{ }^{\circ} \mathrm{C}$. The microchannels were rinsed with $0.2 \mathrm{M} \mathrm{HCl}$ and water and then filled with sieving matrix, 3\% of PVP dissolved in $1 \times \mathrm{TBE}$ buffer with $1 \mathrm{mM}$ YOYO-1. A total of $25 \mu \mathrm{l}$ of sieving matrix solution was added to the buffer reservoir (BR), the sample waste reservoir (SW), and the buffer waste reservoir (BW). The crude PCR product $(25 \mu \mathrm{l})$ diluted in water (1:50) was added to the sample reservoir (SR). The heater plate was mounted on the chip at a $0.4^{\circ}$ slanting angle as described before ${ }^{24}$ and thermostated at $73^{\circ} \mathrm{C}$ to create a $5{ }^{\circ} \mathrm{C}$ temperature gradient from 55 to $60^{\circ} \mathrm{C}$ along the separation channel. During the loading stage, the DNA sample was electrokinetically introduced into the channel by applying the following voltages to the four reservoirs for $80 \mathrm{~s}: \mathrm{BR},+200 \mathrm{~V}$; SR, $0 \mathrm{~V}$; SW, $+400 \mathrm{~V}$; and $\mathrm{BW},+300 \mathrm{~V}$; and during the separation stage: $\mathrm{BR}, 0 \mathrm{~V}$; SR, $+50 \mathrm{~V}$; SW, $+50 \mathrm{~V}$; and $\mathrm{BW}$, $+250 \mathrm{~V}$. Chip-based TGCE assays were performed blindly to detect $\mathrm{K}$-ras mutations in paired stool samples and cancer tissues from these CRC patients and in stool samples from age- and sex-matched controls.

\section{Sensitivity of the Chip-Based TGCE}

Cell lines HT29 and SW480, harboring wild-type K-ras and a homozygous GTT mutant at codon $12,{ }^{27}$ were separately 
cultivated to logarithmic phase. After digestion with trypsin into single-cell suspensions, SW480 cells were pooled at ratios of 1:1, 1:4, 1:16, 1:64, 1:128, 1:256, and 1:512 to realistically simulate tumor heterogeneity. Genomic DNA extraction and amplification of the 293-bp fragments were performed to create target samples for chip-based TGCE. To determine the minimal amount of DNA required for reliable mutation detection, the genomic DNA of 1:1 mixed HT29 and SW480 cells was diluted 250-, 300-, 350-, 400-, 450-, and 500 -fold to simulate low-concentration samples. PCR products amplified from these diluted templates were directly analyzed.

DNA Direct Sequencing and Clone Sequencing Analysis All PCR amplicons of the $K$-ras gene were purified using the DNA purification kit and directly sequenced to confirm the status of codons 12 and 13 using an ABI 377 sequencer. TA clone sequencing was adopted for the samples that showed mutation peak profiles in the electropherograms of chipbased TGCE that were not detectible by direct sequencing.

\section{Analysis of Reproducibility}

To show the reproducibility and robustness of the chip-based TGCE approach, the 50-fold diluted crude PCR product of the stool sample of patient no. 9 was continuously run in replicates for four separation cycles to show the intra-assay variation. The interassay variation was analyzed from four samplings from PCR products of the stool sample of patient no. 9 on four separate TGCE detections in which the sieving matrix was refilled and the temperature gradient was re-formed.

\section{Statistical Analysis}

All statistical analyses employed Fisher's exact test to compare proportions. A two-tailed $P$-value of $<0.05$ was considered statistically significant.

\section{RESULTS}

\section{Melting Calculations for the $\boldsymbol{K}$-ras Gene Region}

Based on the melting predictions, DNA fragments carrying mutations in high-melting domains will not be separated by TGCE because of loss of sequence-dependent migration upon complete strand separation. This problem can be overcome through the introduction of a GC-clamp at one end of the fragment to convert the fragment to a single low-melting domain, ensuring that any mutation present in the target fragment is detectable. ${ }^{16}$ In this study, the Melt 94 program was used to establish the melting map of the 253-bp K-ras gene region (Figure 2). The melting map indicates that codons 12 and $13(+129 \mathrm{bp}$ to $+134 \mathrm{bp})$ lie in the highmelting domain with a $\mathrm{Tm}$ of $\approx 77^{\circ} \mathrm{C}$. The same type of calculation performed for the fragment containing a 40-bp GC-clamp at its $5^{\prime}$ end predicts that the fragment has an additional high-melting domain with a $\mathrm{Tm}$ of $95^{\circ} \mathrm{C}$ at its $5^{\prime}$

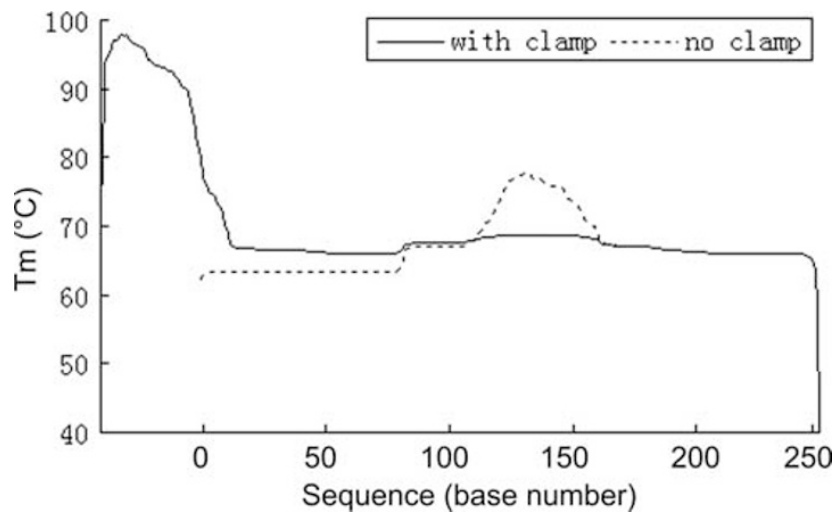

Figure 2 Melting maps for the K-ras gene fragment containing codons 12 and 13. The dashed line is the melting map for the 253-bp fragment, and the solid line is for the 293-bp fragment carrying a 40-bp GC-clamp at its $5^{\prime}$ end.

end, which allows the detection of mutations at codons 12 and 13.

\section{Sensitivity of Chip-Based TGCE}

Under the spatial temperature gradient, a single assay time of 6 min was realized with an extremely low sample consumption of $14 \mathrm{nl}$. Samples with heterozygous mutations will resolve into multiple peaks on the electropherogram that correspond to heteroduplexes and homoduplexes. As shown in Figure 3a, TGCE could identify mutant $K$-ras with an analytical sensitivity as low as $0.2 \%$ (1:512) when the method was applied to a dilution series of SW480 cells exhibiting homozygous $2 \mathrm{G} \rightarrow \mathrm{T}$ mutations in a wild-type HT29 cell background. In comparison, PCR product sequencing required that at least $20 \%$ of the mutant cells be in the wildtype background (Figure 3b). As shown in Figure 4, we found that multi-peaks were clearly resolved for all PCR products amplified from a series of diluted template and that $1 \mu \mathrm{l}$ of 500 -fold diluted initial template DNA (a total of $0.5 \mathrm{ng}$ ) was sufficient for chip-based TGCE analysis.

\section{Detection of Mutant $\boldsymbol{K}$-ras in Clinical Samples}

In this study, 30 paired stool samples and cancer tissues from CRC patients were analyzed using chip-based TGCE and sequencing (Tables 2 and 3). The chip-based TGCE showed that $17(57 \%)$ of the 30 stool samples analyzed contained $K$-ras mutations that were present in $16(53 \%)$ of the corresponding cancer tissues, whereas the direct sequencing only revealed mutations in 6 stools $(20 \%)$ and 10 tissues $(33 \%)$. The former method has a much higher detection rate for the $K$-ras mutation in the stools $(P<0.05)$. Chip-based TGCE disclosed that 1 of the 15 healthy volunteers contained $K$-ras mutations, whereas direct sequencing disclosed none, producing a specificity of 93 and $100 \%$, respectively. No significant difference was noted in the specificities of the two methods (Table 3). Clone sequencing confirmed all positive results of TGCE assay for the patients and volunteers with 

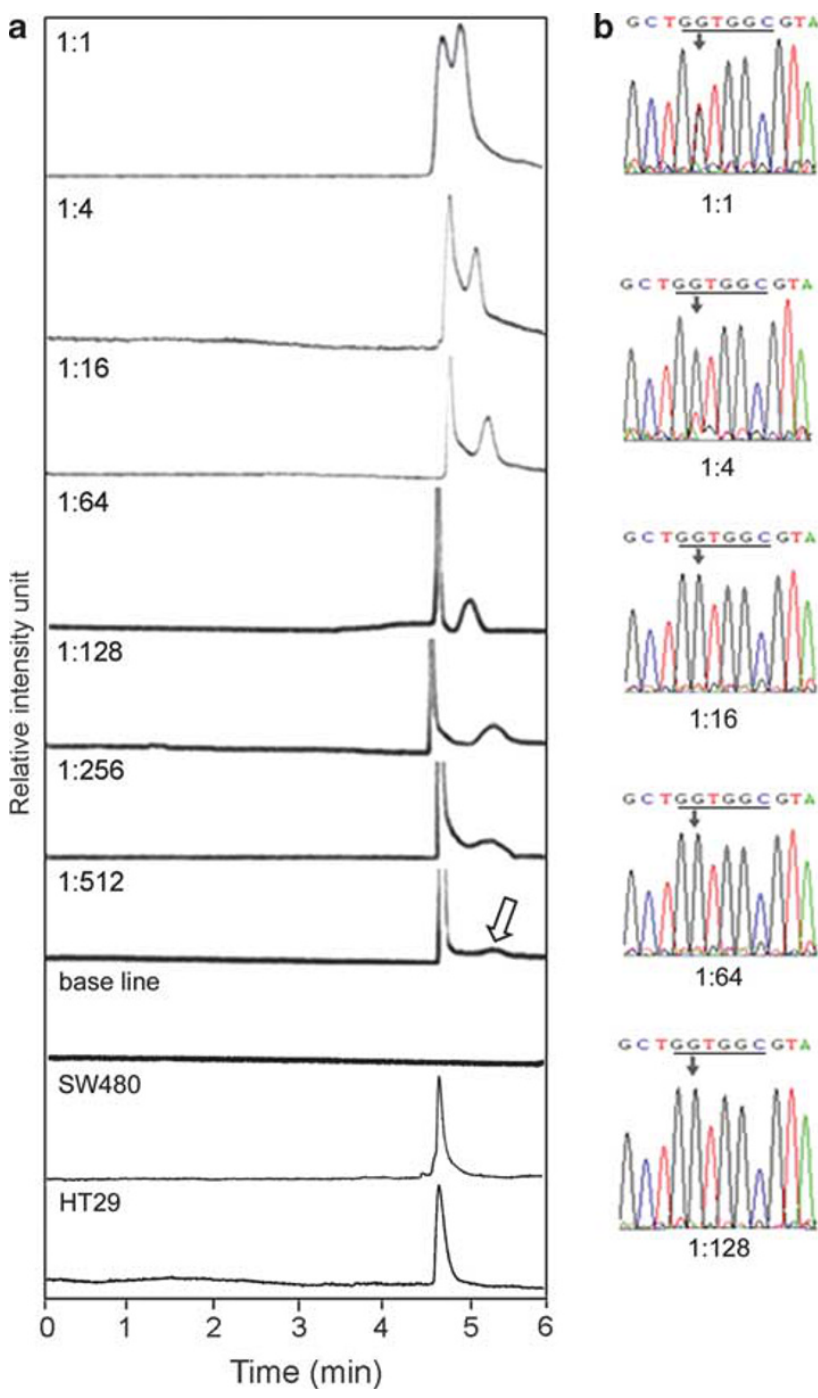

$1: 1$

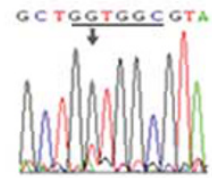

$1: 4$

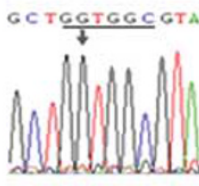

$1: 16$
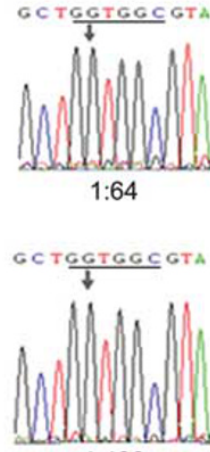

$1: 128$

Figure 3 Detection sensitivity of chip-based TGCE. (a) SW480 cells were diluted with HT29 cells at various ratios. The empty arrow in 1:512 samples indicates the heteroduplex peak. Separated analysis of homozygous mutant SW480 and wild-type HT29 showed a single peak. Electrophoresis was performed with a $5{ }^{\circ} \mathrm{C}$ temperature gradient from 55 to $60^{\circ} \mathrm{C}$, under an electric field of $30 \mathrm{~V} / \mathrm{cm}$. The temperature gradient length was $3 \mathrm{~cm}$. (b) PCR product sequencing data. The underlined bases were codons 12 and 13 (exon 1) of $K$-ras. Heterozygote sites were marked with black arrows. SW480 was mutant homozygote with T (red peak), and HT29 was wild type with G (black peak).

mutant $K$-ras under the sensitivity of direct sequencing. For the CRC patients, $K$-ras mutations at both codons 12 and 13 were detected. Besides the hot spots, mutations at codon 10 in the stool sample and cancer tissue of patient no. 21 were also revealed (Figure 5). Six representative samples with lowabundance $\mathrm{K}$-ras mutations are shown in Figure 5. The lowest mutation proportion was $2 \%$ and occurred in stool sample of patient no. 14 (1/50 clones). For the healthy volunteers, Control-4 who gave a mutant electrophoresis pattern carried a C/T mutation at codon 15 and an $\mathrm{A} / \mathrm{G}$ mutation at codon 32 (Figure 5). The coincidence rate for

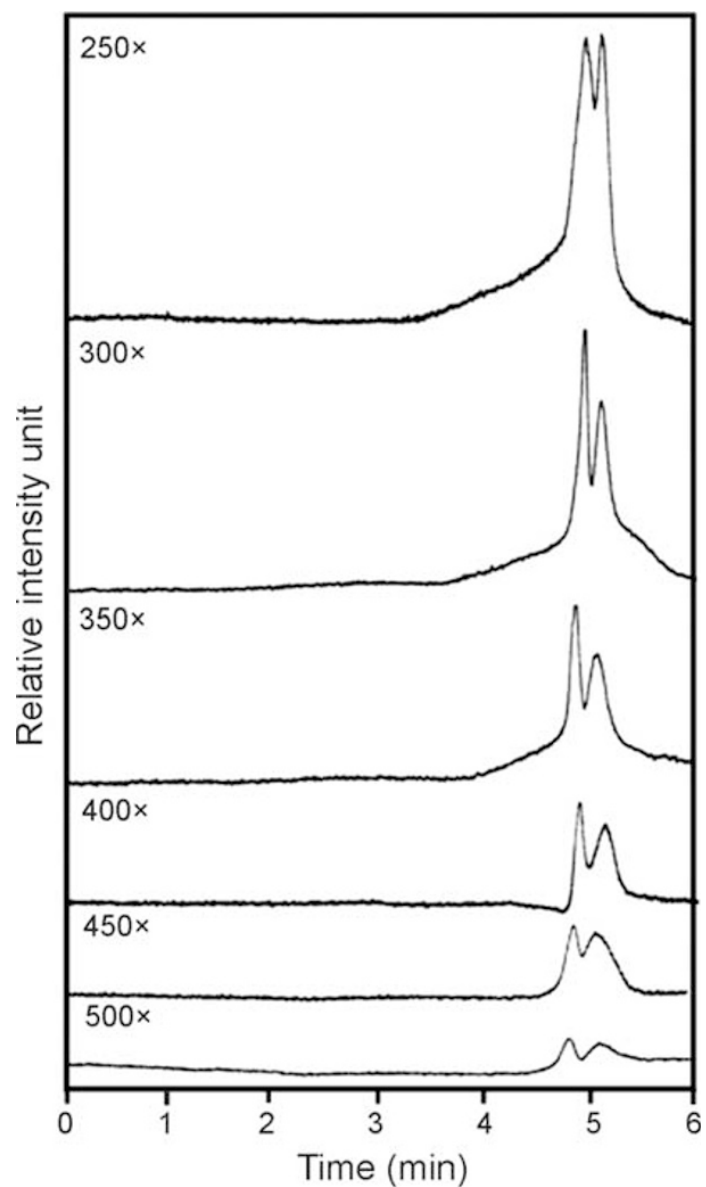

Figure 4 Electropherograms of the $K$-ras gene PCR products amplified from the genomic template of mixed HT29 and SW480 cells in a 1:1 ratio diluted 250-, 300-, 350-, 400-, 450-, or 500-fold with a $5{ }^{\circ} \mathrm{C}$ temperature gradient from 55 to $60^{\circ} \mathrm{C}$ by chip-based TGCE. Other conditions are the same as indicated in Figure 3.

$\mathrm{K}$-ras mutations between stool samples and cancer tissues of CRC patients revealed by chip-based TGCE reached $97 \%$ (29/30). Only patient no. 18 showed a positive stool result but a negative tissue result. The coincidence rate obtained by direct sequencing $(26 / 30,87 \%)$ was a little lower, whereas no significant difference was observed.

\section{Reproducibility}

Four continuous and separate chip-based TGCE detections of PCR products of stool sample of patient no. 9 were performed to demonstrate the assay variations. The recordings showed in Figure 6 demonstrated good intra- and inter-assay variations in peak migration times and peak patterns. The intra- and inter-assay relative standard deviation (RSD) values of migration times were $1.1 \%(n=4)$ and $3.5 \%(n=4)$, respectively. The peak migration time indicates the time for the charged particles to perform directional motion with the action of electric field, which is one of the important parameters for evaluating the reproducibility of electrophoresis. 
Table 2 K-ras mutations in stools and cancers

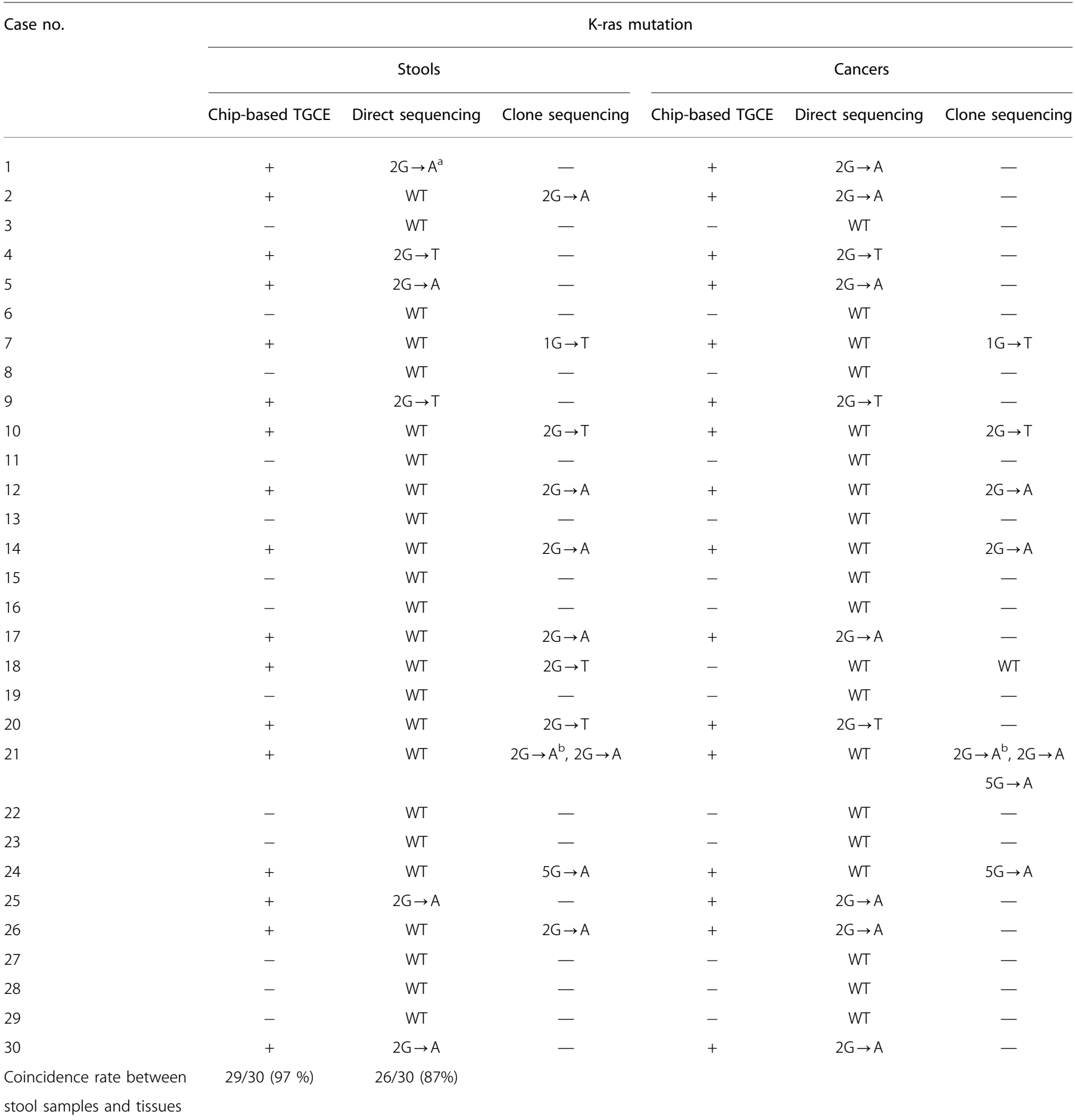

${ }^{\mathrm{a}} 1 \mathrm{G}$ and $2 \mathrm{G}$ indicate first and second bases on codon 12, and $5 \mathrm{G}$ indicates second base on codon 13.

$\mathrm{b}_{2 \mathrm{G}}$ indicates second base on codon 10 .

-, not analyzed.

The symbols (+) and (-) indicate mutant and wild-type $K$-ras detected by chip-based TGCE.

\section{DISCUSSION}

In the past decade, treatment for CRC has significantly improved with the approval of several novel molecular targeting agents, while certain problems are becoming increasingly evident because patients acquire resistance to targeted therapy. It has been reported that mutant $K$-ras provides an 
Table 3 Detection rates and specificities for K-ras mutations of chip-based TGCE and direct sequencing in CRC patients and health volunteers

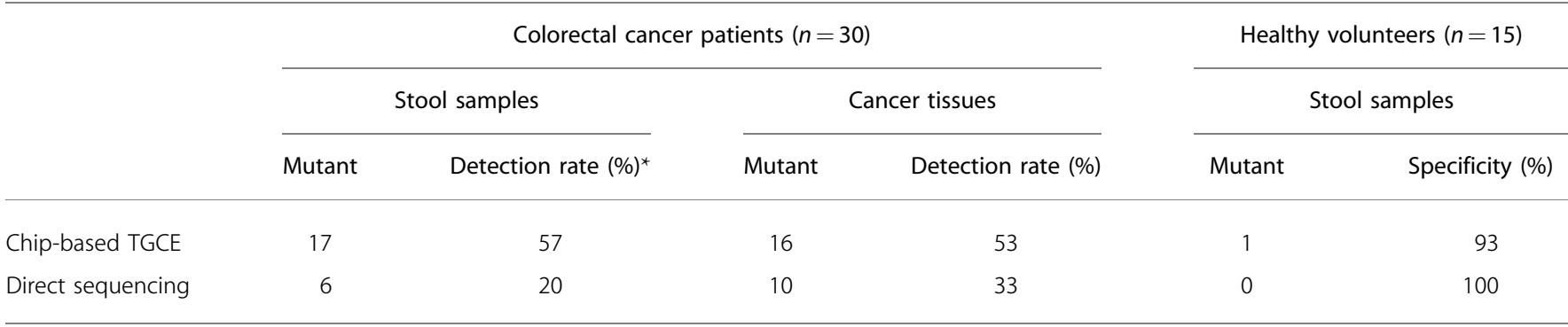

${ }^{*} P<0.05$.

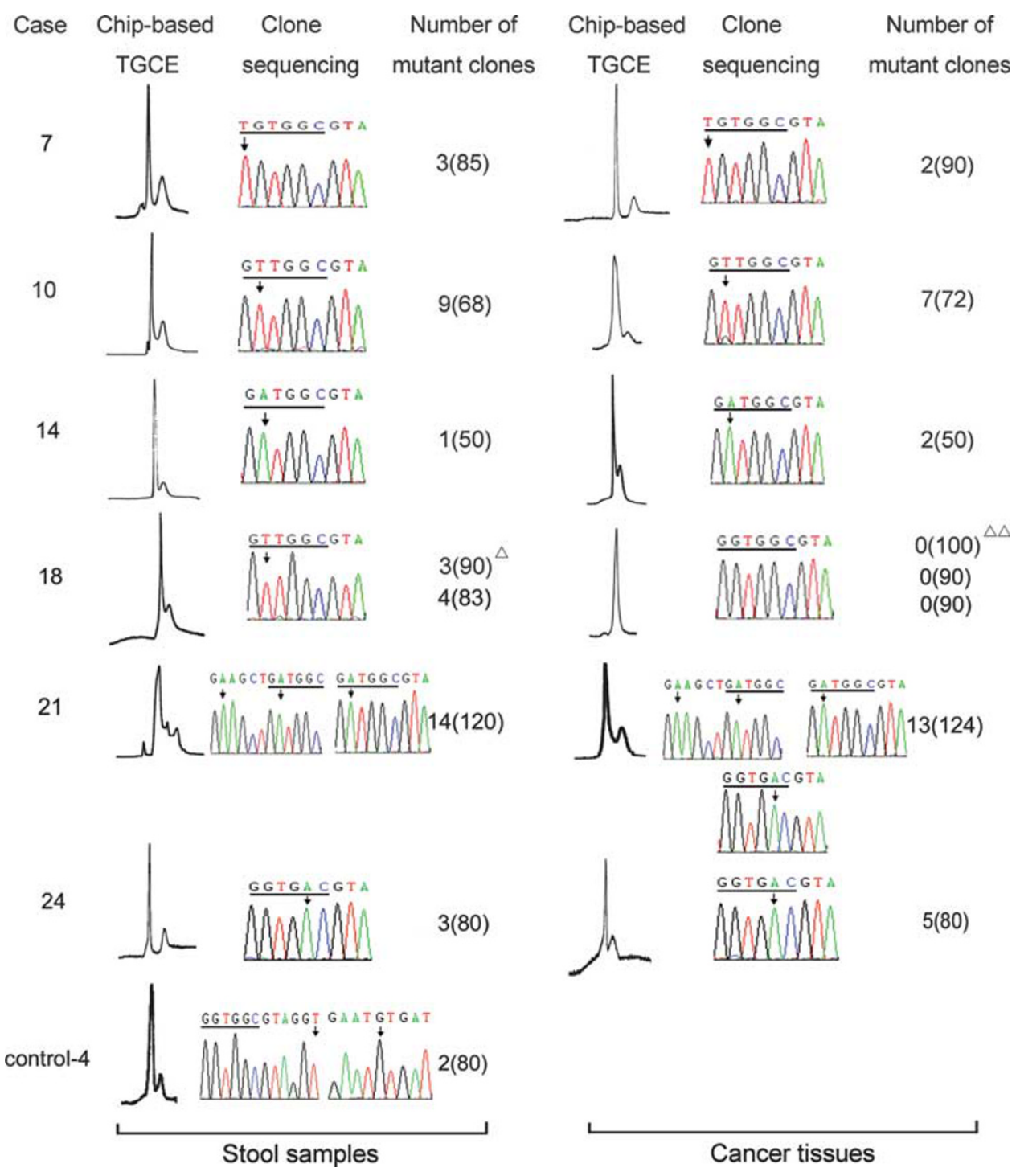

Figure 5 Representative K-ras gene mutation-positive stool samples and cancer tissues detected by chip-based TGCE but not by direct sequencing. Mutant sites are marked by black arrows. Codons 12 and 13 are underlined with solid lines. ${ }^{\triangle}$ Two aliquots of the stool sample of patient no. 18 were detected individually. ${ }^{\triangle \triangle}$ Three parts of the cancer tissues of patient no. 18 were detected individually.

independent negative predictive marker for EGFR-targeted therapy in CRC; thus, several methods have been established to screen $K$-ras gene to achieve successful personalized medicine. ${ }^{4-6}$ Based on the good clinical compliance and the findings of several research groups that revealed $K$-ras mutations in stools consistent with the $K$-ras status in matching 

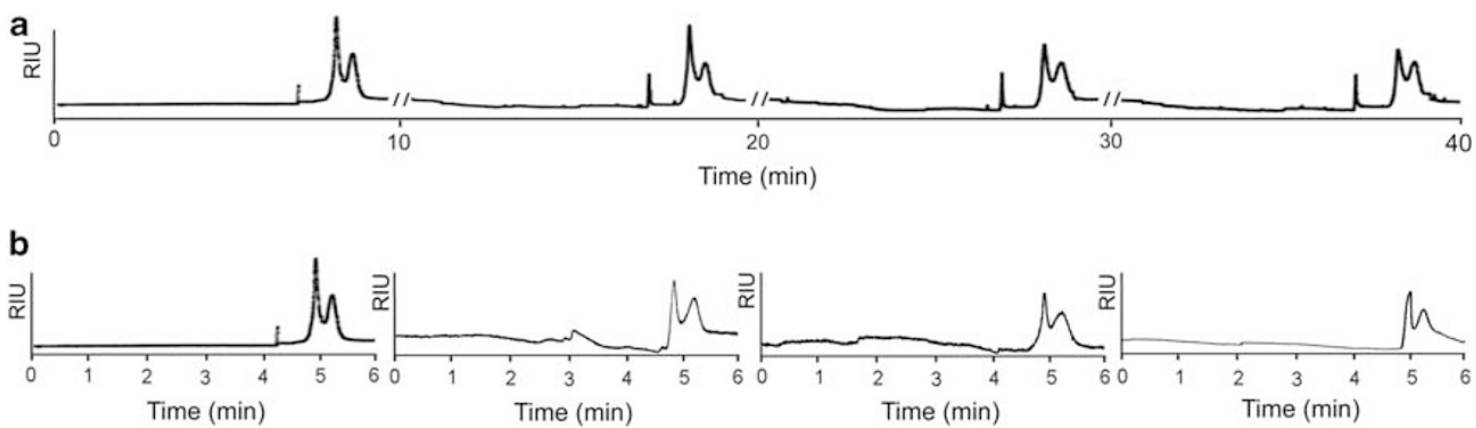

Figure 6 Reproducibility analysis of the stool sample from patient no. 9 employing a temperature gradient of $55-60^{\circ} \mathrm{C}$. (a) Intra-reproducibility and (b) inter-reproducibility. RIU: relative intensity unit. Other conditions are the same as indicated in Figure 3.

tumor tissues, ${ }^{28-30}$ mutated DNA in stools has become the latest target of several detection methods. ${ }^{8-10}$ Although stoolbased DNA detection could report CRC tissue K-ras genotypes faithfully, severe difficulties because of complicated procedures and low sensitivity have hampered further progress in using stool samples to detect $K$-ras mutations and preventatively screen for CRC.

In this work, highly sensitive and rapid detection of $K$-ras mutation was achieved using chip-based TGCE. This assay could reliably detect as low as $0.2 \%$ mutant $K$-ras in a background of wild-type genomic DNA and trace PCR product amplified from $0.5 \mathrm{ng}$ of template, suggesting that this method is capable of detecting low-abundance $K$-ras mutations in stool samples. The results were much better than those obtained by $\mathrm{Li}$ et $a$, $^{31}$ who adopted standard TGCE to detect a model mutant DNA sample diluted with the corresponding wild-type fragment at a ratio of 1:9. As reported by Bian et al, ${ }^{20}$ although limiting-dilution PCR (LD-PCR) followed by TGCE significantly improved the detection of detectable mutations to $1 \%$, the remarkably increased numbers of PCR reactions made this work highly uneconomical and time consuming. Mutant allele-specific amplification and mutant-enriched PCR could achieve a very high sensitivity $(0.01 \%) .{ }^{32,33}$ However, the former lacks enough specificity when used to detect gene mutations because the amplification of wild-type will proceed if there is a slight change in conditions; the latter has certain disadvantages of taking time for several consecutive amplifications and introducing mutations during these PCRs. ${ }^{13,34}$ Digital melt curve (DMC), a newly developed screening method, showed a satisfactory scanning ability for low-abundance mutations $(0.1 \%)$ in the informative regions of target genes, ${ }^{12}$ whereas it was expensive at present for use in clinical molecular diagnostic purposes. Time-cost was another important aspect to evaluate the screening method. In the conventional slab gel electrophoresis, several separate steps including gel preparation, sample loading, separation, staining, and photographic imaging will take $>2 \mathrm{~h}$, whereas on the microfluidic platform all the steps mentioned above can be integrated together to perform on-line detection, resulting in the increased speed. In this study, the whole automated chip electrophoresis could be accomplished within $6 \mathrm{~min}$.
In this study, the K-ras status from 30 paired stool samples and cancer tissues was detectable using chip-based TGCE, and the lowest percentage of the mutant $K$-ras was $2 \%$ in the stool sample of patient no. 14. The detection rate of $K$-ras mutation in stool specimens from the CRC patients was $57 \%$, which was slightly higher than that noticed in cancer tissues (53\%). Currently, the majority of studies have shown that mutations are found more frequently in tissues than in stools. $^{13,29,30,35,36}$ In this study, four patients (nos. 2, 17, 20, and 26) exhibiting mutant electrophoresis patterns in both stool samples and cancer tissues were found by direct sequencing to carry $K$-ras mutations in their cancer tissues but not in their stool samples, whereas clone sequencing confirmed the K-ras mutations in their stool samples, showing that the proportions of mutant $K$-ras were 9, 12, 15 , and $13 \%$, respectively, which demonstrated the lower abundance of $\mathrm{K}$-ras mutations in stool samples compared with that in cancer tissues. As described in many papers, methods with high sensitivities could achieve high mutation detection rates. $^{12,32,33,37}$ Marchetti et al ${ }^{37}$ compared direct sequencing with mutation-enriched sequencing in 90 colorectal carcinomas. The prevalence of tumors harboring $\mathrm{K}$-ras mutations was $40 \%$ using direct sequencing and 55\% using mutationenriched sequencing, which had a higher sensitivity. ${ }^{37}$ In our study, K-ras mutations were identified in $57 \%$ of the 30 stool samples by chip-based TGCE, significantly higher than that obtained by direct sequencing. Thus, the high stool-based mutation detection rate can be attributable to the high sensitivity of the technique. Simultaneously, with the increase of sensitivity, the coincidence rate for K-ras mutations between stool samples and cancer tissues was also improved. An up to 97\% agreement of K-ras mutations was observed between stool samples and cancer tissues from our chip-based TGCE method, which demonstrated that stool DNA-based assays could provide accurate information about CRC patients by using more sensitive methods. In this study, only patient no. 18 presented a positive stool result but a negative tissue result for the K-ras mutation. To further confirm the results, $K$-ras mutations in two more areas of the cancer tissue and in another aliquot of the stool sample of patient no. 18 were analyzed by chip-based TGCE and confirmed by clone 
sequencing. As shown in Figure 5, no mutation was revealed in the cancer tissues, but the stool sample still harbored mutant $K$-ras. This discordance was probably because of heterogeneity within the same tumor and contaminations of diet or intestinal flora and other gastrointestinal tumors or nonneoplastic conditions such as pancreatic hyperplasia. ${ }^{38-41}$ The protocols of the QIAamp DNA Stool mini kit used here had reduced the ratio of nonhuman DNA as far as possible. In this study, only one healthy volunteer was found to contain K-ras mutations by chip-based TGCE. No significant difference was shown in the specificity of chip-based method and direct sequencing, which indicated that the increased sensitivity did not affect the specificity.

Compared with conventional TGCE, the higher sensitivity of the chip-based TGCE method can be attributed to several factors. First, TGCE relies on the electrophoretic separation of homoduplexes and heteroduplexes in the sieving matrix under a given electric field. The high separation efficiency can be obtained by applying high field strength; however, high field strength may produce high joule heating in conventional $\mathrm{CE}$ because of a lower ratio of channel surface area to channel volume, resulting in the reduction of separation efficiency. In contrast, if the ratio of surface area to the volume of the channel of the chip-based CE is higher, joule heating is released easily, and higher field can be used to achieve improved resolution, which in turn leads to an excellent separation of homoduplexes and heteroduplexes in TGCE. ${ }^{42,43}$ Second, according to the separation principle of TGCE, the resolution efficiency for mutant DNA fragments is dependent on the temperature melting profile of the detected sequences. If the mutant sequence is located in the lowmelting domain in a whole DNA fragment, the migration differences between heteroduplexes and homoduplexes during $\mathrm{CE}$ are readily produced and retained. The mutant codons 12 and 13 are in the relatively high-melting domain in the 253-bp fragments of the K-ras gene, and a GC-clamp is introduced to the fragment to form a high-melting domain relative to mutant base sites. It has been reported that TGCE combined with the GC-clamp technique is capable of achieving nearly $100 \%$ mutation detection efficiency. ${ }^{18}$ Without the GC-clamp, the 'nonclamped' 253-bp fragments differing by a base change at codon 12 or 13 will not be separated due to the loss of sequence-dependent migration because of complete strand separation. Third, the sensitivity of TGCE is in large part determined by the temperature profile. In this study, an established slantwise radiative heating system is used to produce a stable and uniform spatial temperature gradient along the separation channel by simply slanting a thermostated aluminum heating plate on the chip surface using a thin spacer at the inlet of the separation channel, with a precision of $0.1{ }^{\circ} \mathrm{C} .{ }^{24}$ The novel property of the heating system ensures good CE separation efficiency and reproducibility. In this study the migration time precisions for the intra- and inter-assay reached 1.1 and $3.5 \%$ RSD. Finally, the sensitive LIF detector is applied combined with a new type of fluorescent dye, YOYO-1. YOYO-1 is essentially nonfluorescent in the absence of nucleic acids and exhibits a 100- to 1000 -fold increase in fluorescence upon binding to DNA; therefore, this system can detect as low as $0.5 \mathrm{ng} / \mathrm{ml}$ of double-stranded DNA (dsDNA). ${ }^{44,45}$ In contrast, the fluorescence of the commonly used $\mathrm{EtBr}$ is only enhanced $\sim 20$ - to 30 -fold after intercalation into dsDNA, which requires a relatively high concentration of DNA sample to be effective in the TGCE assay $(50-500 \mathrm{ng} / \mu \mathrm{l})$. Meanwhile, unconjugated EtBr itself will cause a high-fluorescence background and interferes with the detection sensitivity. ${ }^{23,44,45}$

Although our preliminary clinical investigation is limited because of restricted access to more CRC samples, the results of our study showed that the detection accuracy rate reached $100 \%$ after verification by clone sequencing. The high accuracy might be due to the relatively high sensitivity of this method, because the mutations harbored in stools can be as little as $1 \%$ in general, ${ }^{11}$ which is much higher than the threshold concentration $(0.2 \%)$ of this chip-based TGCE method. From our results, $K$-ras mutations and base substitutions in stool samples were identical to that in cancer tissues. One of the most significant advantages of TGCE was the ability to detect virtually any base alteration within a gene sequence. In this study, this advantage meant that it could detect mutations at both codons 12 and 13 , which were proved to be predictors guiding EGFR-targeted therapy. In this study we observed this phenomenon that mutations at codons 12 and 13 in different samples and within the same sample (cancer tissue of patient no. 21) were both revealed, exhibiting a wide applicability of this assay. Meanwhile, mutations outside codons 12 and 13 could be also revealed according to the basic principle of TGCE, ${ }^{16}$ such as a G/A mutation at codon 10 in the stool sample and cancer tissue of patient no. 21, and a C/T mutation at codon 15 and an A/G mutation at codon 32 in the stool sample of Control-4. Although these mutations were disclosed, the specificity of chip-based TGCE was still up to $93 \%$. As a screening method, the results still should be further confirmed by subsequent sequencing of the gene.

A cost analysis of this process is also critical for chip-based TGCE to become a mutation detection technique, involving chip fabrication and reagents to perform the analysis. In this work, each glass chip costs $\sim 2.0$ USD, and the cost can be drastically reduced by using polymer materials to perform batch fabrication in the future, matching the requirements for clinical practice such as low-cost, disposability, and individualized detection. An extremely low sample consumption of $14 \mathrm{nl}$ for each analysis was obtained. Furthermore, reagent costs for chip-based TGCE analysis were also quite low because of microscaling, even if the fluorescent dye YOYO-1 was applied. For just the chip-based TGCE portion of the assay, the reagent cost per sample is $\sim 0.02$ USD.

In conclusion, we developed a highly sensitive and fast chip-based TGCE method for detecting low-abundant gene 
mutations. The applicability of the method was demonstrated by the successful noninvasive detection of $\mathrm{K}$-ras mutation within stool samples of the CRC patients, which facilitated the therapeutic evaluation of mutations for further personalized therapy. In view of its technological superiority, the chip-based TGCE method exhibits a high potential for more comprehensive application. A large number of different DNA fragments can be detected by TGCE as long as the electrophoresis is performed under an enough wide temperature gradient range to cover their $\mathrm{Tm}$ values. In this study the chip-based TGCE platform has been proven to establish a stable and uniform $10^{\circ} \mathrm{C}$ temperature gradient and achieve mutation detections of various DNA fragments. ${ }^{24}$ Therefore, a panel of target genes associated with specific cancers, including some large genes with widely dispersed potential mutational sites, can be analyzed simultaneously by further developing continuous sample introduction system or increasing the number of parallel channels on the platform, so that the high-throughput detection can be realized.

\section{ACKNOWLEDGEMENT}

This work was supported by the National Natural Science Foundation of China (Grant nos. 20875130, 20375047, and 30901727) and grants from the Ministry of Education of the Liaoning province of China (Grant no. 2006097).

\section{DISCLOSURE/CONFLICT OF INTEREST}

The authors declare no conflict of interest.

1. Jiang Y, Kimchi ET, Staveley-O'Carroll KF, et al. Assessment of K-ras mutation: a step toward personalized medicine for patients with colorectal cancer. Cancer 2009;115:3609-3617.

2. Eng C. The evolving role of monoclonal antibodies in colorectal cancer: early presumptions and impact on clinical trial development Oncologist 2010;15:73-84.

3. Ma WW, Adjei AA. Novel agents on the horizon for cancer therapy. CA Cancer J Clin 2009;59:111-137.

4. Karapetis CS, Khambata-Ford S, Jonker DJ, et al. K-ras mutations and benefit from cetuximab in advanced colorectal cancer. N Engl J Med 2008;359:1757-1765.

5. Amado RG, Wolf $M$, Peeters $M$, et al. Wild-type KRAS is required for panitumumab efficacy in patients with metastatic colorectal cancer. J Clin Oncol 2008;26:1626-1634.

6. Waldner MJ, Neurath MF. The molecular therapy of colorectal cancer. Mol Aspects Med 2010;31:171-178.

7. Allegra CJ, Jessup JM, Somerfield MR, et al. American Society of Clinical Oncology provisional clinical opinion: testing for KRAS gene mutations in patients with metastatic colorectal carcinoma to predict response to anti-epidermal growth factor receptor monoclonal antibody therapy. J Clin Oncol 2009;27:2091-2096.

8. Osborn NK, Ahlquist DA. Stool screening for colorectal cancer: molecular approaches. Gastroenterology 2005;128:192-206.

9. Levin B, Lieberman DA, McFarland B, et al. Screening and surveillance for the early detection of colorectal cancer and adenomatous polyps, 2008: a joint guideline from the American Cancer Society, the US MultiSociety Task Force on Colorectal Cancer, and the American College of Radiology. CA Cancer J Clin 2008;58:130-160.

10. Chung DC. Stool DNA testing and colon cancer prevention: another step forward. Ann Intern Med 2008;149:509-510.

11. Hasegawa $Y$, Takeda S, Ichii S, et al. Detection of K-ras mutations in DNAs isolated from feces of patients with colorectal colorectal tumors by mutant-allele-specific amplification (MASA). Oncogene 1995;10: 1441-1445.
12. Zou H, Taylor WR, Harrington JJ, et al. High detection rates of colorectal neoplasia by stool DNA testing with a novel digital melt curve assay. Gastroenterology 2009;136:459-470.

13. Nishikawa T, Maemura K, Hirata I, et al. A simple method of detecting $\mathrm{K}$-ras point mutations in stool samples for colorectal cancer screening using one-step polymerase chain reaction/restriction fragment length polymorphism analysis. Clin Chim Acta 2002;318:107-112.

14. Notarnicola M, Cavallini A, Cardone R, et al. K-ras and p53 mutations in DNA extracted from colonic epithelial cells exfoliated in faeces of patients with colorectal cancer. Dig Liver Dis 2000;32:131-136.

15. Ho-Pun-Cheung $A$, Choblet $S$, Choblet $S$, et al. Detection of single nucleotide polymorphisms by minisequencing on a polypyrrole DNA chip designed for medical diagnosis. Lab invest 2006;86:304-313.

16. Murphy KM, Berg KD. Mutation and single nucleotide polymorphism detection using temperature gradient capillary electrophoresis. Expert Rev Mol Diagn 2003:3:811-818.

17. Gelfi C, Righetti PG, Cremonesi L, et al. Detection of point mutations by capillary electrophoresis in liquid polymers in temporal thermal gradients. Electrophoresis 1994;15:1506-1511.

18. Sheffield VC, Cox DR, Lerman LS, et al. Attachment of a 40-base-pair $\mathrm{G}+\mathrm{C}$-rich sequence (GC-clamp) to genomic DNA fragments by the polymerase chain reaction results in improved detection of single-base changes. Proc Natl Acad Sci USA 1989;86:232-236.

19. Bertina RM, Koeleman BP, Koster $\mathrm{T}$, et al. Mutation in blood coagulation factor $\mathrm{V}$ associated with resistance to activated protein C. Nature 1994;369:64-67.

20. Bian Y, Matsubayashi H, Li CP, et al. Detecting low-abundance p16 and p53 mutations in pancreatic juice using a novel assay: heteroduplex analysis of limiting dilution PCRs. Cancer Biol Ther 2006;5: 1392-1399.

21. Kleparník K, Bocek P. DNA diagnostics by capillary electrophoresis. Chem Rev 2007;107:5279-5317.

22. Ohno K, Tachikawa K, Manz A. Microfluidics: applications for analytical purposes in chemistry and biochemistry. Electrophoresis 2008;29: 4443-4453.

23. Buch JS, Kimball C, Rosenberger $F$, et al. DNA mutation detection in a polymer microfluidic network using temperature gradient gel electrophoresis. Anal Chem 2004;76:874-881.

24. Zhang HD, Zhou J, Xu ZR, et al. DNA mutation detection with chipbased temperature gradient capillary electrophoresis using a slantwise radiative heating system. Lab Chip 2007;7:1162-1170.

25. Jia ZJ, Fang Q, Fang ZL. Bonding of glass microfluidic chips at room temperatures. Anal Chem 2004;76:5597-5602.

26. Fang $\mathrm{Q}, \mathrm{Xu} G \mathrm{G}$, Fang ZL. A high-throughput continuous sample introduction interface for microfluidic chip-based capillary electrophoresis systems. Anal Chem 2002;74:1223-1231.

27. Gayet J, Zhou XP, Duval A, et al. Extensive characterization of genetic alterations in a series of human colorectal cancer cell lines. Oncogene 2001;20:5025-5032.

28. Sidransky D, Tokino T, Hamilton SR, et al. Identification of ras oncogene mutations in the stool of patients with curable colorectal tumor. Science 1992;256:102-105.

29. Nollau P, Moser C, Weinland G, et al. Detection of K-ras mutations in stools of patients with colorectal cancer by mutation-enriched PCR. Int J Cancer 1996;66:332-336.

30. Lev Z, Kislitsin D, Rennert G, et al. Utilization of K-ras mutations identified in stool DNA for the early detection of colorectal cancer. J Cell Biochem Suppl 2000;34:35-39.

31. Li Q, Liu Z, Monroe H, et al. Integrated platform for detection of DNA sequence variants using capillary array electrophoresis. Electrophoresis 2002;23:1499-1511.

32. Tada M, Omata M, Kawai $S$, et al. Detection of ras gene mutations in pancreatic juice and peripheral blood of patients with pancreatic adenocarcinoma. Journal Title: Cancer Res 1993;53:2472-2474.

33. Kahn SM, Jiang W, Culbertson TA, et al. Rapid and sensitive nonradioactive detection of mutant K-ras genes via 'enriched' PCR amplification. Oncogene 1991;6:1079-1083.

34. Kwok S, Kellogg DE, McKinney $\mathrm{N}$, et al. Effects of primer-template mismatches on the polymerase chain reaction: human immunodeficiency virus type 1 model studies. Nucleic Acids Res 1990; 18:999-1005.

35. Ito $\mathrm{Y}$, Kobayashi $\mathrm{S}$, Taniguchi $\mathrm{T}$, et al. Frequent detection of K-ras mutation in stool samples of colorectal carcinoma patients after 
improved DNA extraction: comparison with tissue samples. Int J Oncol 2002;20:1263-1268.

36. Mixich $F$, loana $M$, Voinea $F$, et al. Noninvasive detection through REMS-PCR technique of K-ras mutations in stool DNA of patients with colorectal cancer. J Gastrointestin Liver Dis 2007;16:5-10.

37. Marchetti A, Gasparini G. K-ras mutations and cetuximab in colorectal cancer. N Engl J Med 2009;360:833-834.

38. Yamashita N, Minamoto T, Ochiai A, et al. Frequent and characteristic K-ras activation and absence of p53 protein accumulation in aberrant crypt foci of the colon. Gastroenterology 1995;108: 434-440.

39. Otori K, Oda Y, Sugiyama K, et al. High frequency of K-ras mutations in human colorectal hyperplastic polyps. Gut 1997;40:660-663.

40. Klaassen $\mathrm{CH}$, Jeunink MA, Prinsen $\mathrm{CF}$, et al. Quantification of human DNA in feces as a diagnostic test for the presence of colorectal cancer. Clin Chem 2003;49:1185-1187.
41. Haug U, Wente $M N$, Seiler $C M$, et al. Stool testing for the early detection of pancreatic cancer: rationale and current evidence. Expert Rev Mol Diagn 2008;8:753-759.

42. Petersen NJ, Nikolajsen RP, Mogensen KB, et al. Effect of Joule heating on efficiency and performance for microchip-based and capillary-based electrophoretic separation systems: a closer look. Electrophoresis 2004;25:253-269.

43. Evenhuis CJ, Haddad PR. Joule heating effects and the experimental determination of temperature during CE. Electrophoresis 2009;30: 897-909.

44. Benson SC, Mathies RA, Glazer AN. Heterodimeric DNA-binding dyes designed for energy transfer: stability and applications of the DNA complexes. Nucleic Acids Res 1993;21:5720-5726.

45. Benson SC, Mathies RA, Glazer AN. Heterodimeric DNA-binding dyes designed for energy transfer: stability and applications of the DNA complexes. Nucleic Acids Res 1993;21:5727-5735. 